The Chinese Society of Theoretical and Applied Mechanics

Chinese Journal of Mechanics Press, Beijing, China

Allerton Press, INC., New York, U.S.A.

\title{
AN ANALYSIS OF SUBGRID-RESOLVED SCALE INTERACTIONS WITH USE OF RESULTS FROM DIRECT NUMERICAL SIMULATIONS
}

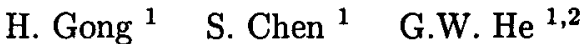 \\ ${ }^{1}$ (Environment, Safety and Health Division and Center for Nonlinear Studies \\ Los Alamos National Laboratory, Los Alamos, NM 87545, USA) \\ ${ }^{2}$ (LNM, Institute of Mechanics, Chinese Academy of Sciences, \\ Beijing 100080, China)
}

\begin{abstract}
Subgrid nonlinear interaction and energy transfer are analyzed using direct numerical simulations of isotropic turbulence. Influences of cutoff wave number at different ranges of scale on the energetics and dynamics have been investigated. It is observed that subgrid-subgrid interaction dominates the turbulent dynamics when cut-off wave number locates in the energy-containing range while resolvedsubgrid interaction dominates if it is in the dissipation range. By decomposing the subgrid energy transfer and nonlinear interaction into 'forward' and 'backward' groups according to the sign of triadic interaction, we find that individually each group has very large contribution, but the net of them is much smaller, implying that tremendous cancellation happens between these two groups.
\end{abstract}

KEY WORDS: subgrid nonlinear interaction, energy transfer, large eddy simulation (LES), subgrid scale (SGS), direct numerical simulation, isotropic turbulence

\section{INTRODUCTION}

In large eddy simulation (LES), the subgrid scale (SGS) is modeled to reflect the effects of the filtered subgrid scale on the resolved scale. Although considerable progress has been made in developing and improving SGS models, there is still not a commonly accepted model. Testing of SGS models conducted by Clark et al. ${ }^{[1]}$ revealed that prediction of the subgrid scale stress by most existing models is generally poor. Apparently a better understanding of the physical process of subgrid scale nonlinear interactions is necessary for improvements of the existing SGS models.

Analysis of energy transfer between resolved and subgrid scales in isotropic turbulence were performed by Domaradzki et al. ${ }^{[2]}$ They calculated the exact eddy viscosity and compared it to the value predicted by Kraichnan's theoretical model ${ }^{[3]}$. More detailed analysis on the locality of energy transfer when cutoff wave number is in the inertial and dissipation ranges have been done by Domaradzki and Rogallo[4], Zhou ${ }^{[5]}$, and Brasseur and Wei ${ }^{[6]}$. Investigation of the subgrid energy transfer in physical space has also been conducted by Domaradzki et al. ${ }^{[7]}$ and Kerr and Domaradzki ${ }^{[8]}$. 
Energy transfer is a principal physical process influencing the evolution of turbulent fields. Understanding this process is crucial to designing a better SGS model in LES. Although many aspects of this process have been studied previously by noted researchers, there are still some issues remaining unclear. One of them is the analysis of the energy transfer if the cutoff wave number moves from inertial range to the energy-containing range. So far all studies are limited to that with the cutoff wave number located in the inertial or dissipation range. But in practical application of LES, the length scale used for the filter could be greater than the scale for inertial range and reside in the energy-containing range. It is most likely to occur when the simulated flow field is wall-bounded and the grid size near the wall may be relatively large enough to enter into the energy containing range. The solution to this difficulty in LES is to use a damping function to correct the length scale. The damping function is an ad hoc function and purely empirical. For a better model to account for the near wall effect, further analysis is needed for subgrid nonlinear interaction with cutoff wave number in the energy-containing range.

Another issue related to the energy transfer is the phenomenon of energy backscatter which is widely recognized since the work of Leith ${ }^{[9]}$. Prediction of the energy backscatter should be a indispensable property of a successful SGS model. Backscatter is the result of energy transfer from smaller scales to larger scales. Basically, energy transfer between scales takes place bidirectionally, namely, forward and backward transfers. If the net of forward and backward transfer is negative then we say that energy backscatter happens. Since the forward and backward transfer are two basic components of the net transfer, it is both fundamental and useful to analyze them separately.

In this work the net subgrid energy transfer along with its forward and backward components are investigated when cutoff wave number moves from dissipation and inertial range to energy-containing range using velocity fields based on direct numerical simulations of isotropic turbulence.

\section{BASIC QUANTITIES}

\subsection{Basic Equations}

For homogeneous turbulence, the incompressible Navier-Stokes equation in spectral space are

$$
\frac{\partial}{\partial t} u_{i}(k)=-\nu k^{2} u_{i}(k)+N_{i}(k)
$$

where, $u_{i}(k)$ is the velocity components and $N_{i}(k)$ is the nonlinear term given by

$$
\begin{gathered}
N_{i}(\boldsymbol{k})=\sum_{\boldsymbol{k}=\boldsymbol{p}+\boldsymbol{q}} N_{i}(\boldsymbol{k} \mid \boldsymbol{p}, \boldsymbol{q}) \\
N_{i}(\boldsymbol{k} \mid \boldsymbol{p}, \boldsymbol{q})=-\frac{\mathrm{i}}{2} P_{i l m}(\boldsymbol{k}) u_{l}(\boldsymbol{p}) u_{m}(\boldsymbol{q})
\end{gathered}
$$

where $P_{i l m}(k)$ in the following represents a tensor accounting for pressure and incompressibility

$$
P_{i l m}(k)=k_{m}\left(\delta_{i l}-\frac{k_{i} k_{l}}{k^{2}}\right)+k_{l}\left(\delta_{i m}-\frac{k_{i} k_{m}}{k^{2}}\right)
$$

Energy equation in spectral space can be derived from the Navier-Stokes equation as

$$
\frac{\partial}{\partial t} e(k)=-\nu k^{2} e(k)+T(k)
$$


where, the kinetic energy $e(k)$ and energy transfer $T(k)$ are defined as follows

$$
\begin{aligned}
& e(k)=\frac{1}{2} u_{i}(k) u_{i}^{*}(k) \\
& T(k)=\sum_{k=p+q} T(k \mid p, q) \\
& T(k \mid p, q)=-\frac{1}{2} \operatorname{Im}\left\{P_{i l m}(k) u_{i}^{*}(k) u_{l}(p) u_{m}(q)\right\}
\end{aligned}
$$

where 'Im' means imaginary part and ' $*$ ' indicates the operation of complex conjugate. Energy spectrum $E(k)$ and energy transfer spectrum $T(k)$ are calculated by summing up $e(k)$ and $T(k)$ over the wavenumber shell $k$

$$
\begin{aligned}
E(k) & =\sum_{k-0.5<|k| \leq k+0.5} e(k) \\
T(k) & =\sum_{k-0.5<|k| \leq k+0.5} T(k)
\end{aligned}
$$

\subsection{Equations for Resolved Scales}

Introducing the cutoff wavenumber $k_{\mathrm{c}}$, which is evidently associated with the width of filter, one can define the resolved and subgrid scales as

$$
k= \begin{cases}k_{\mathrm{r}} & \text { if }|k| \leq k_{\mathrm{c}} \\ k_{\mathrm{s}} & \text { if }|k|>k_{\mathrm{c}}\end{cases}
$$

where subscriptions 's' and ' $r$ ' represent subgrid scale and resolved scale, respectively.

Taking Fourier transformation of Eq.(1) and then applying sharp cutoff filtering to it leads to an momentum equation for resolved velocity: $u_{\mathrm{r} i}\left(\boldsymbol{k}_{\mathrm{r}}\right)$

$$
\frac{\partial}{\partial t} u_{\mathrm{r} i}\left(k_{\mathrm{r}}\right)=-\nu k_{\mathrm{r}}^{2} u_{\mathrm{r} i}\left(k_{\mathrm{r}}\right)+N_{\mathrm{ri}}\left(k_{\mathrm{r}}\right)+N_{\mathrm{s} i}\left(k_{\mathrm{r}}\right)
$$

where $N_{\mathrm{ri}}\left(\boldsymbol{k}_{\mathrm{r}}\right)$ is the resolved nonlinear term and $N_{\mathrm{si}}\left(\boldsymbol{k}_{\mathrm{r}}\right)$ is the subgrid nonlinear term, viz

$$
\begin{aligned}
N_{\mathrm{ri}}\left(k_{\mathrm{r}}\right) & =\sum_{k_{\mathrm{r}}=p_{\mathrm{r}}+q_{\mathrm{r}}} N_{i}\left(k_{\mathrm{r}} \mid p_{\mathrm{r}}, q_{\mathrm{r}}\right) \\
N_{\mathrm{s} i}\left(k_{\mathrm{r}}\right)= & =\underbrace{2 \sum_{k_{\mathrm{r}}=p_{\mathrm{r}}+q_{\mathrm{s}}} N_{i}\left(k_{\mathrm{r}} \mid p_{\mathrm{r}}, q_{\mathrm{s}}\right)}_{N_{\mathrm{sri}}\left(k_{\mathrm{r}}\right)}+\underbrace{\sum_{k_{\mathrm{r}}=p_{\mathrm{s}}+q_{\mathrm{s}}} N_{i}\left(k_{\mathrm{r}} \mid p_{\mathrm{s}}, q_{\mathrm{s}}\right)}_{N_{\mathrm{ssi}}\left(k_{\mathrm{r}}\right)}
\end{aligned}
$$

Equation of kinetic energy for the resolved scale is similarly derived by Eq.(5)

$$
\frac{\partial}{\partial t} e\left(k_{\mathrm{r}}\right)=-\nu k_{\mathrm{r}}^{2} e\left(k_{\mathrm{r}}\right)+T_{\mathrm{r}}\left(k_{\mathrm{r}}\right)+T_{\mathrm{s}}\left(\boldsymbol{k}_{\mathbf{r}}\right)
$$

where $T_{\mathrm{r}}\left(k_{\mathrm{r}}\right)$ is the energy transfer due to the interaction between resolved scales and $T_{\mathrm{s}}\left(k_{\mathrm{r}}\right)$ is the subgrid energy transfer due to the interaction involving subgrid scales.

$$
T_{\mathrm{r}}\left(\boldsymbol{k}_{\mathrm{r}}\right)=\sum_{\boldsymbol{k}_{\mathrm{r}}=\boldsymbol{p}_{\mathrm{r}}+\boldsymbol{q}_{\mathrm{r}}} T\left(\boldsymbol{k}_{\mathrm{r}} \mid \boldsymbol{p}_{\mathrm{r}}, \boldsymbol{q}_{\mathrm{r}}\right)
$$




$$
T_{\mathrm{s}}\left(k_{\mathrm{r}}\right)=\underbrace{2 \sum_{k_{\mathrm{r}}=p_{\mathrm{r}}+q_{\mathrm{s}}} T\left(k_{\mathrm{r}} \mid \boldsymbol{p}_{\mathrm{r}}, \boldsymbol{q}_{\mathrm{s}}\right)}_{T_{\mathrm{sr}}\left(k_{\mathrm{r}}\right)}+\underbrace{\sum_{k_{\mathrm{r}}=p_{\mathrm{s}}+q_{\mathrm{s}}} T\left(k_{\mathrm{r}} \mid p_{\mathrm{s}}, \boldsymbol{q}_{\mathrm{s}}\right)}_{T_{\mathrm{sz}}\left(\boldsymbol{k}_{\mathrm{r}}\right)}
$$

In the expressions of subgrid nonlinear term $N_{\mathrm{s} i}\left(k_{\mathrm{r}}\right)$ and energy transfer $T_{\mathrm{s}}\left(\boldsymbol{k}_{\mathrm{r}}\right)$, we separated the contribution of resolved-subgrid interaction $\left(N_{\mathrm{sri}}\left(k_{\mathrm{r}}\right), T_{\mathrm{sr}}\left(k_{\mathrm{r}}\right)\right)$ and that of subgridsubgrid interaction $\left(N_{\mathrm{ssi}}\left(k_{\mathrm{r}}\right), T_{\mathrm{sr}}\left(k_{\mathrm{r}}\right)\right)$. Thus, we are able to examine the relative contribution of each component to the total when cutoff wavenumber is in different scale ranges.

\subsection{Decomposition of Subgrid Energy Transfer and Nonlinear Term}

From Eq.(17) it is clear that subgrid energy transfer $T_{\mathbf{B}}\left(k_{\mathrm{r}}\right)$ represents the summation of the results due to the nonlinear interactions of modes $\boldsymbol{p}_{\mathrm{r}}$ and $\boldsymbol{q}_{\mathrm{s}}$ (or $\boldsymbol{p}_{\mathrm{s}}$ and $\boldsymbol{q}_{\mathrm{s}}$ ) forming a triad with $k_{\mathrm{r}}$. Among all the triadic interaction, some of them result in energy transfer to mode $\boldsymbol{k}_{\mathrm{r}}$ from subgrid scale when $T\left(\boldsymbol{k}_{\mathrm{r}} \mid \boldsymbol{p}_{\mathrm{r}}, \boldsymbol{q}_{\mathrm{s}}\right)>0$ (or $T\left(\boldsymbol{k}_{\mathrm{r}} \mid \boldsymbol{p}_{\mathrm{s}}, \boldsymbol{q}_{\mathrm{s}}\right)>0$ ), are called 'backward' transfer. Other triadic interactions cause the energy transfer from mode $k_{\mathrm{r}}$ to subgrid scale when $T\left(k_{\mathrm{r}} \mid \boldsymbol{p}_{\mathrm{r}}, \boldsymbol{q}_{\mathrm{s}}\right)<0$ (or $T\left(\boldsymbol{k}_{\mathrm{r}} \mid \boldsymbol{p}_{\mathrm{s}}, \boldsymbol{q}_{\mathrm{s}}\right)<0$ ), are called 'forward' transfer. According to this observation $T_{\mathrm{s}}\left(k_{\mathrm{r}}\right)$ can be decomposed into forward and backward transfer parts

$$
T_{\mathrm{s}}\left(k_{\mathrm{r}}\right)=T_{\mathrm{s}}^{+}\left(k_{\mathrm{r}}\right)+T_{\mathrm{s}}^{-}\left(k_{\mathrm{r}}\right)
$$

where

$$
\begin{aligned}
& T_{\mathrm{s}}^{+}\left(\boldsymbol{k}_{\mathrm{r}}\right)=\sum_{\boldsymbol{k}_{\mathrm{r}}=\boldsymbol{p}_{\mathrm{s}}+\boldsymbol{q}_{\mathrm{s}}}\left[T\left(\boldsymbol{k}_{\mathrm{r}} \mid \boldsymbol{p}_{\mathrm{s}}, \boldsymbol{q}_{\mathrm{s}}\right)<0\right]+2 \sum_{\boldsymbol{k}_{\mathrm{r}}=\boldsymbol{p}_{\mathrm{r}}+\boldsymbol{q}_{\mathrm{s}}}\left[T\left(\boldsymbol{k}_{\mathrm{r}} \mid \boldsymbol{p}_{\mathrm{r}}, \boldsymbol{q}_{\mathrm{s}}\right)<0\right] \\
& T_{\mathrm{s}}^{-}\left(\boldsymbol{k}_{\mathrm{r}}\right)=\sum_{\boldsymbol{k}_{\mathrm{r}}=\boldsymbol{p}_{\mathrm{s}}+\boldsymbol{q}_{\mathrm{s}}}\left[T\left(\boldsymbol{k}_{\mathrm{r}} \mid \boldsymbol{p}_{\mathrm{s}}, \boldsymbol{q}_{\mathrm{s}}\right)>0\right]+2 \sum_{\boldsymbol{k}_{\mathrm{r}}=\boldsymbol{p}_{\mathrm{r}}+\boldsymbol{q}_{\mathrm{s}}}\left[T\left(\boldsymbol{k}_{\mathrm{r}} \mid \boldsymbol{p}_{\mathrm{r}}, \boldsymbol{q}_{\mathrm{s}}\right)>0\right]
\end{aligned}
$$

\section{NUMERICAL SIMULATION}

The $3 D$ turbulent velocity field used for this analysis are obtained from direct numerical simulations of the Navier-Stokes equations using the standard pseudo-spectra method. Direct numerical simulation of decaying flows has been performed using a random initial condition with a prescribed energy spectrum. $256^{3}$ grids are generated for computation to meet the demand of accuracy and the initial Reynolds number is $R_{\lambda}=113$. The velocity field of decaying flows used in the analysis is taken at time $t=8$, in eddy turnover units. The Reynolds number at that time is $R_{\lambda}=32$. The energy peak is at wave number $k=4$ and the entropy peak at $k=14$. In this study, the subgrid-resolved scale dynamics were analyzed when cutoff wave number locates at $k_{\mathrm{c}}=5, k_{\mathrm{c}}=10$, and $k_{\mathrm{c}}=20$. These cutoff wave numbers reside in the energy-containing, inertial, and dissipation ranges respectively in decaying turbulence.

\section{RESULTS AND DISCUSSION}

\subsection{Energy Transfer}

Energy transfer through resolved scales are shown in Fig.1 with different cutoff wave numbers. The total energy transfer $T\left(k_{\mathrm{r}}\right)$ is plotted with its components, resolved transfer $T_{\mathrm{r}}\left(k_{\mathrm{r}}\right)$ and subgrid transfer $T_{s}\left(k_{\mathrm{r}}\right)$. It can be noted that total energy flux is dominated by the contribution from the subgrid energy transfer if cutoff wave number is in the energy 
containing range (Fig.1(a)), but the energy transfer due to the interaction between resolved scales contributes more when cutoff moves to inertial range (Fig.1(b)). If the cutoff is in dissipation range, the resolved transfer dominates the total transfer up to $k=0.5 k_{\mathrm{c}}$ while both resolved and subgrid transfers contribute equally at the cutoff vicinity (Fig.1(c)). These results suggest that a SGS model is not important when cutoff is in the dissipation range because the total energy flux is dominated by the resolved scale interactions for the most part of energy containing region. But modeling of the subgrid energy transfer is important when cutoff is located in the inertial range. When cutoff moves into energy containing range, it is crucial for a SGS model to correctly predict the subgrid energy flux.

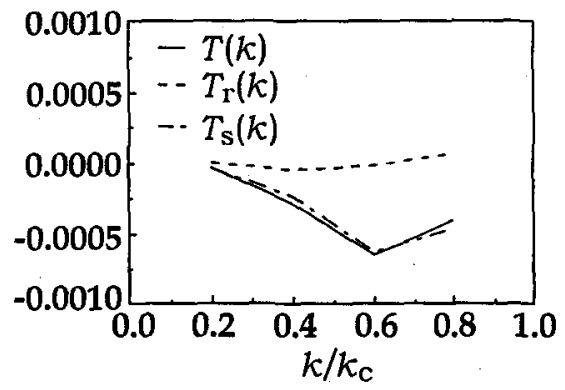

(a) $k_{\mathrm{c}}=5$

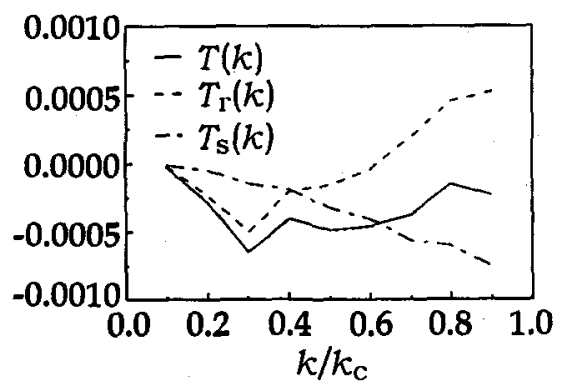

(b) $k_{\mathrm{c}}=10$

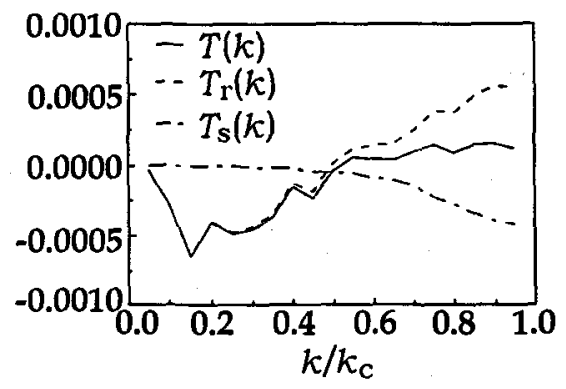

(c) $k_{\mathrm{c}}=20$

Fig.1 Energy transfers for decaying flow

Relationship between the subgrid energy transfer $T_{\mathrm{s}}\left(k_{\mathrm{r}}\right)$ and its decompositions, $T_{\mathrm{ss}}\left(k_{\mathrm{r}}\right)$ and $T_{\mathrm{sr}}\left(k_{\mathrm{r}}\right)$, are illustrated in Fig.2. When $k_{\mathrm{c}}$ is in the dissipation range(Fig.2(c)), the contribution to $T_{\mathrm{s}}\left(k_{\mathrm{r}}\right)$ is almost wholely from $T_{\mathrm{sr}}\left(k_{\mathrm{r}}\right)$, indicating the energy transfer due to the interaction between subgrid scales $T_{\mathrm{ss}}\left(k_{\mathrm{r}}\right)$ is negligible. If $k_{\mathrm{c}}=10$, which is in the inertial range, both $T_{\mathrm{ss}}\left(k_{\mathrm{r}}\right)$ and $T_{\mathrm{sr}}\left(k_{\mathrm{r}}\right)$ contribute to the total subgrid transfer $T_{\mathrm{s}}\left(k_{\mathrm{r}}\right)$. From Fig.2(b), it is found that $T_{\mathrm{ss}}\left(k_{\mathrm{r}}\right)$ contributes more than $T_{\mathrm{sr}}\left(k_{\mathrm{r}}\right)$ when $k<0.6 k_{\mathrm{c}}$ and vice versa when $k>0.6 k_{\mathrm{c}}$. If $k_{\mathrm{c}}$ is in the energy-containing range, it should be noted from Fig.2(a) that $T_{\mathrm{ss}}\left(k_{r}\right)$ dominates the subgrid transfer $T_{\mathrm{s}}\left(k_{\mathrm{r}}\right)$.

In Fourier space, the averaged energy equation (5) for resolved scales is closed by a subgrid-scale modeling. In an eddy-viscosity type approach, the subgrid energy transfer can be written as

$$
T_{\mathrm{s}}\left(k_{\mathrm{r}}\right)=-2 \nu_{t}\left(k_{\mathrm{r}}\right) k_{\mathrm{r}}^{2} E\left(k_{\mathrm{r}}\right)
$$


by which eddy viscosity $\nu_{t}$ is determined and normalized as follows

$$
\nu_{t}^{*}=\frac{\nu_{t}\left(k_{\mathrm{r}}\right)}{\left[E\left(k_{\mathrm{c}}\right) / k_{\mathrm{c}}\right]^{1 / 2}}
$$

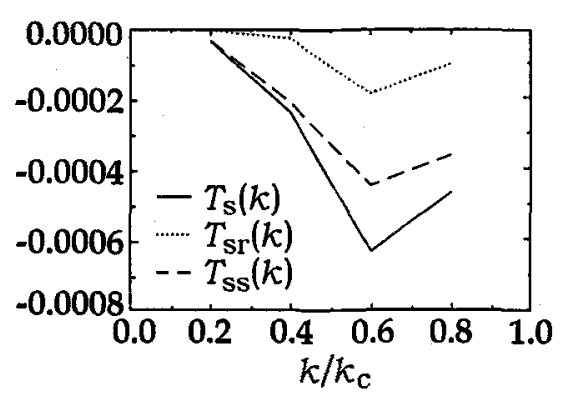

(a) $k_{\mathrm{c}}=5$

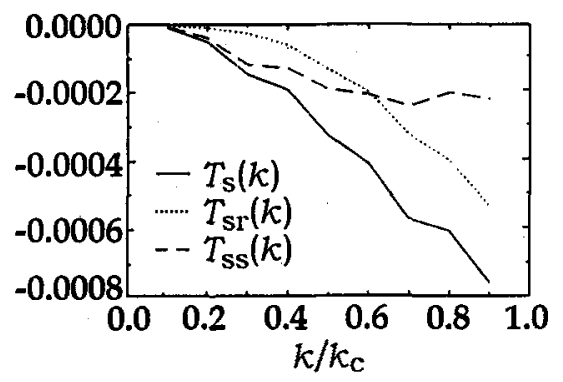

(b) $k_{\mathrm{c}}=10$

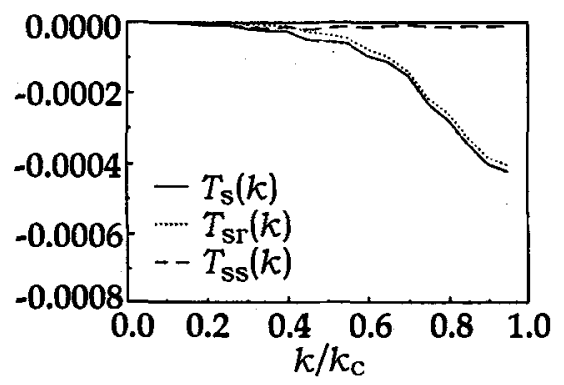

(c) $k_{\mathrm{c}}=20$

Fig.2 Subgrid energy transfer for decaying flows

The calculated eddy viscosities $\nu_{t}^{*}$ by direct numerical simulation are shown in Fig.3(a). When $k_{\mathrm{c}}=20$, the viscosity increases in the vicinity of the cutoff wave number $k_{\mathrm{c}}$, exhibiting the importance of local interactions. Eddy viscosity becomes larger as the cutoff wave number moves into inertial and energy-containing ranges, indicating the importance of nonlocal interactions.

\subsection{Forward and Backward Energy Transfer}

Forward and backward subgrid energy transfers $T_{s}^{+}\left(k_{\mathrm{r}}\right)$ and $T_{s}^{-}\left(k_{\mathrm{r}}\right)$ are defined by Eqs.(19) and (20). The sum of these two part of transfer results in the net transfer. The normalized eddy viscosities representing forward and backward transfers are calculated and compared with the total eddy viscosity. Figure 3(b) shows the comparison for $k_{\mathrm{c}}=10$. It is obvious that the magnitudes of both forward and backward components are much larger than that of the total one. The forward and backward parts are almost balanced with a much smaller difference. Similar results are also found when cutoff is in other scale ranges, implying that most energy transfer to and from resolved scales almost cancel each other with much less net energy transfer. This observed relation among the total energy transfer and its forward and backward components may suggest that modeling the forward and backward parts individually instead of the net transfer alone is not a practical approach. 


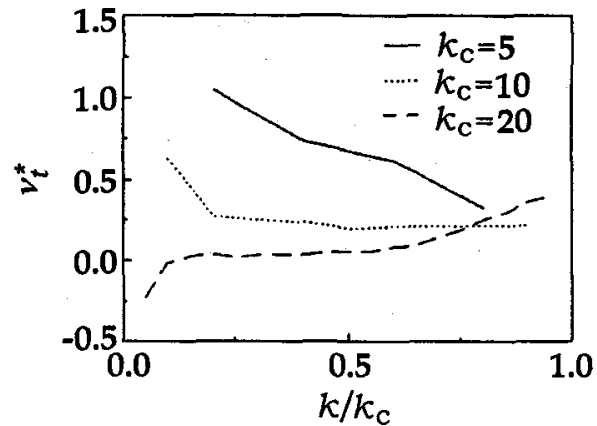

(a) Eddy viscosity calculated from direct numerical simulation

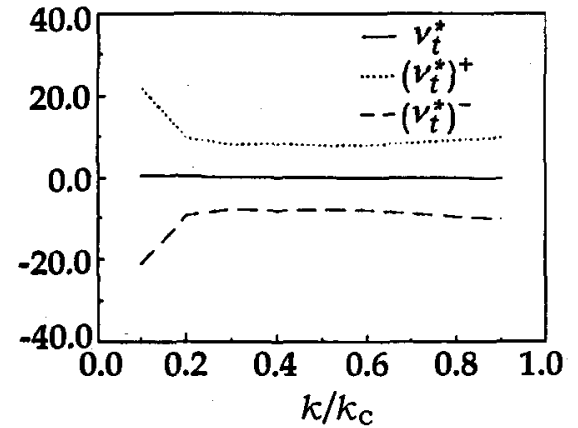

(b) Eddy viscosity with its forward and backward components

Fig.3

\section{CONCLUSIONS}

Cutoff wavenumber is the maximal resolved motion in numerical simulation. Different cutoff wavenumbers affect the energetics and dynamics of isotropic turbulence in LES. In this paper, an analysis of the effects of different cutoff wave number on the energetics and dynamics of isotropic turbulence has been performed by using velocity fields obtained from direct numerical simulation. The cutoff wave number has been chosen such that it locates at three different scale ranges: energy-containing range, inertial range and dissipation range.

Our analysis reveals that when the cutoff wave number is in the energy containing range, the subgrid energy transfer is significant and extends to the whole resolved scales. The subgrid-subgrid scale interaction dominates the energetics and dynamics. When cutoff is located in the inertial range, both subgrid-subgrid and resolved-subgrid scale interactions contribute to the energetics and dynamics with the former contributing more at lower wave number and less in the vicinity of the cutoff. If cutoff moves to the dissipation range, resolved-subgrid scale interaction dominates but it is only important in the vicinity of the cutoff. These features of the relationship between subgrid scale interactions may suggest that it could be useful to parameterize the subgrid-subgrid and resolved-subgrid interactions separately.

The 'forward' and 'backward' dynamics has been investigated by decomposing the subgrid energy transfer into a 'forward' part and a 'backward' part according to the sign of triadic interactions. It is found that each component individually has very large contribution, but the net is much smaller. This observation implies that there are tremendous cancellations among 'forward' and 'backward' interactions. It appears that it may not be useful to model 'forward' and 'backward' dynamics separately.

Acknowledgement This work was supported by Department of Energy at Los Alamos National Laboratory. The numerical computations were performed at the Advanced Computing Laboratory at Los Alamos National Laboratory.

\section{REFERENCES}

1 Clark RA, Ferziger JH, Reynolds WC. Evaluation of subgrid-scale models using an accurately simulated turbulent flow. J Fluid Mech, 1979, 91(1): 1 16 
2 Domaradzki JA, Metcalfe RW, Rogallo RS, Riley JJ. Analysis of subgrid-scale eddy viscosity with use of results from direct numerical simulations. Phys Rev Lett, 1987, 58(6): 547 550

3 Kraichnan RH. Eddy viscosity in two and three dimensions. J Atmos Sci, 1976, 38: 2747

4 Domaradzki JA, Rogallo SS. Local energy transfer and nonlocal interactions in homogeneous, isotropic turbulence. Phys Fluids A, 1990, 2(3): 413 426

5 Zhou Y. Degrees of locality of energy transfer in the inertial range. Phys Fluids A, 1993, 5(5): $1092 \sim 1094$

6 Brasseur JG, Wei C. Interscale dynamics and local isotropy in high Reynolds number turbulence within triadic interactions. Phys Fluids, 1994, 6(2): 842 870

7 Domaradzki JA, Liu W, Brachet ME. An analysis of subgrid-scale interactions in numerically simulated isotropic turbulence. Phys Fluids A, 1993, 5(7): 1747 1759

8 Kerr RM, Domaradzki JA. Small-scale properties of nonlinear interactions and subgrid-scale energy transfer in isotropic turbulence. Phys Fluids, 1996, 5(7): 197 208.

9 Leith CE. Stochastic backscatter in a subgrid-scale mode: plane shear mixing layer. Phys Fluids $A, 1990,2(3): 297 \sim 299$ 